\title{
Biotypes of Haemophilus parainfluenzae from the respiratory secretions in chronic bronchitis
}

\author{
DIANA C. TAYLOR*t, A. W. CRIPPS $\ddagger$, R. L. CLANCY*, K. MURREE-ALLEN§, M. J. HENSLEY*, \\ N. A. SAUNDERS* and D. C. SUTHERLANDT \\ - Faculty of Medicine, The University of Newcast/e, $\uparrow$ Auspharm Institute for Mucosal Immunology, Hunter Technology \\ Centre, Rankin Drive, Short/and, $¥$ Hunter Area Pathology Service, Newcastle, §Department of Thoracic Medicine and \\ THunter Immunology Unit, Royal Newcastle Hospital, Newcastle, New South Wales, Australia
}

\begin{abstract}
Summary. A total of 2401 isolates of Haemophilus parainfluenzae was isolated from respiratory secretions of 36 healthy adults and 128 patients with chronic bronchitis over a period of 1 year. The isolates were allocated to eight biotypes, by their production of indole, urease and ornithine decarboxylase. Biotypes I and II constituted most of the isolates of $H$. parainfluenzae from the oropharynx of controls ( $75 \%$ ) and chronic bronchitics $(c .90 \%)$. Among the patients, there was no difference in the isolation rate between oropharyngeal swabs and sputum specimens. Biotypes III, IV, VI, VII and VIII were isolated less frequently, as was a new taxon defined here as bioptype $\mathrm{V}$ which does not produce indole, urease or ornithine decarboxylase. Biotype III was isolated significantly less frequently from cases of chronic bronchitis than from controls, whereas biotype II was isolated somewhat more frequently from the patients, especially during acute episodes.
\end{abstract}

\section{Introduction}

In recent years Haemophilus parainfluenzae has been recognised as both a normal inhabitant and an opportunist pathogen of the upper respiratory tract (URT). It is thought that dental, oropharyngeal, pulmonary or middle ear infections frequently precede systemic infections caused by this organism. ${ }^{1}$ The most commonly reported infection is endocarditis ${ }^{1,2}$ although cases of pneumonia, ${ }^{3,4}$ septic arthritis ${ }^{4}$ and bacteraemia ${ }^{5}$ have been reported. Frederiksen and $\mathrm{Kilian}^{6}$ have argued that reports of meningitis and epiglottitis caused by $H$. parainfluenzae represent misidentified strains of $H$. influenzae. ${ }^{7} H$. parainfluenzae constitutes at least $74 \%$ of the isolates of Haemophilus spp. from the pharynx $;{ }^{8}$ it is commonly isolated from the URT and lower respiratory tract (LRT) secretions of chronic bronchitics but is generally considered to be non-pathogenic. However, there is accumulating evidence to suggest that this organism may cause disease of a similar spectrum to that of non-typable $H$. influenzae in the LRT. Rhind et al. ${ }^{9}$ presented evidence that $H$. parainfluenzae was a pathogen in respiratory tract infections, and other investigators ${ }^{10-12}$ have demonstrated a high isolation rate in sputum specimens. Studies have shown that $H$. parainfluenzae slows ciliary activity in the respiratory tract. ${ }^{13}$ This may

Received 24 July 1990; revised version accepted 26 June 1991. ‡Correspondence should be sent to Dr A. W. Cripps, Hunter Area Pathology Service, Locked Bag 1, Newcastle Mail Centre, Newcastle, NSW 2310, Australia. contribute to diminished mucociliary clearance of micro-organisms and, possibly, chronic bronchial sepsis. ${ }^{11}$ With its high isolation rate in respiratory secretions and its increasing implication as an opportunist pathogen, emphasis has been placed on the importance of characterising isolates of $\mathrm{H}$. parainfluenzae from the respiratory secretions in chronic bronchitis.

Over a 1-year period, we investigated the distribution of biotypes of $H$.parainfluenzae in the URT and LRT of chronic bronchitic patients, some apparently free of infection and others with acute exacerbations; and we compared these strains with URT isolates from age-matched healthy adults.

\section{Materials and methods}

\section{Patients and healthy controls}

We recruited 148 patients from a hospital outpatient clinic to participate in a prospective study of chronic bronchitis, defined according to American Thoracic Society criteria $;{ }^{14}$ median age 66 , range $18-84$ years. An age-matched control group of 36 subjects with no recognised lung disease were also studied; median age 66 , range $48-75$ years. Whereas $44 \%$ of the chronic bronchitic subjects smoked, only $11 \%$ of the controls smoked. Oropharyngeal swabs were taken from both groups monthly for 1 year. Samples of sputum were collected from the bronchitic subjects at the same time when possible. The sputum specimens were checked 
by Gram's stain and microscopy; by the criteria of Courcol et al., ${ }^{15}$ those with greater than four squamous epithelial cells per low power field were rejected. Episodes of acute bronchitis were defined as "production of increased volume and purulence of sputum". Routine samples were taken from patients who had no episode of acute bronchitis for at least 21 days. Cultures of $H$. parainfluenzae were isolated from 128 of the patients and from all 36 control subjects.

\section{Bacterial culture and identification}

Specimens were incubated overnight at $37^{\circ} \mathrm{C}$, in $\mathrm{CO}_{2} 5 \%$ in air, on chocolate agar (defibrinated horse blood $5 \%$ ) containing bacitracin (Sigma) $300 \mu \mathrm{g} / \mathrm{ml}$. Up to four morphologically distinct colonies were selected from each sample. Small gram-negative pleomorphic coccobacilli from a single colony were subcultured to chocolate agar and incubated overnight. The V-factor requirement was assessed by the observation of satellite growth beside a streak of the Oxford strain of Staphylococcus aureus on blood agar, on which the presence or absence of haemolysis was noted also. The $\mathrm{X}$-factor requirement was deduced from ability to metabolise $\partial$-aminolaevulinic acid and produce porphyrins. ${ }^{16}$

\section{Biochemical tests}

Isolates that were $\mathrm{V}$-dependent and $\mathrm{X}$-independent were biotyped by their production of indole, urease and ornithine decarboxylase ${ }^{17}$ with the Minitek Differentiation System (BBL Microbiology Systems). ${ }^{18,19}$ Tests were read after incubation for $4 \mathrm{~h}$ at $37^{\circ} \mathrm{C}$.

Isolates that gave negative results in these three biochemical tests were further characterised, with the Minitek system, by their production of acid from glucose, sucrose, lactose, xylose, mannose, mannitol and galactose. ${ }^{17}$ In triplicate tests on each strain, no discrepancies were found.

To test for catalase production, a small amount of the culture was removed with a clean sterile glass rod and emulsified in a drop of $\mathrm{H}_{2} \mathrm{O}_{2}$ solution on a glass slide; the drop was observed for effervescence.

To test for oxidase production by Kovacs' method, ${ }^{20}$ a portion of a colony was removed with a sterile glass rod and rubbed on a strip of filter paper impregnated with a freshly prepared solution of tetramethyl-pphenylenediamine dihydrochloride $1 \%$; oxidase-positive colonies gave a deep purple colour within $10 \mathrm{~s}$.

\section{Results}

By their production of indole, urease and ornithine decarboxylase, we differentiated 2401 isolates of $H$. parainfluenzae into eight biotypes (table I). The distribution of these biotypes in controls and chronic bronchitic patients is shown in table II. The isolates were predominantly of biotypes I and II. From the oropharynx, biotype I was isolated at a similar frequency from the control group $(43 \%)$ and the chronic bronchitic group ( $47 \%$ ). Biotype II constituted $32 \%$ and $42 \%$ of the oropharyngeal isolates, respectively. Biotype III was isolated less frequently-16\% and $7 \%$, respectively. Biotypes I, II and III had similar

Table I. Differentiation of biotypes of $H$. parainfluenzae by tests for three metabolic products

\begin{tabular}{l|ccc}
\hline & \multicolumn{3}{|c}{ Result of test for production of } \\
Biotype* & $\begin{array}{l}\text { indole from } \\
\text { tryptophan }\end{array}$ & urease & $\begin{array}{c}\text { ornithine } \\
\text { decarboxylase }\end{array}$ \\
\cline { 2 - 4 } I & - & - & + \\
II & - & + & + \\
III & - & + & - \\
IV & + & + & + \\
V & - & - & - \\
VI & + & + & - \\
VII & + & - & - \\
VIII & + & & + \\
\hline
\end{tabular}

* Biotype $\mathrm{V}$ is defined in the present study. The other seven have been described previously: biotypes I-III $;^{17}$ biotype IV $;^{5}$ biotypes VI and VII $;^{26}$ biotype VIII. ${ }^{25}$

Table II. Biotypes of $H$. parainfluenzae isolated from chronic bronchitic patients and from healthy controls

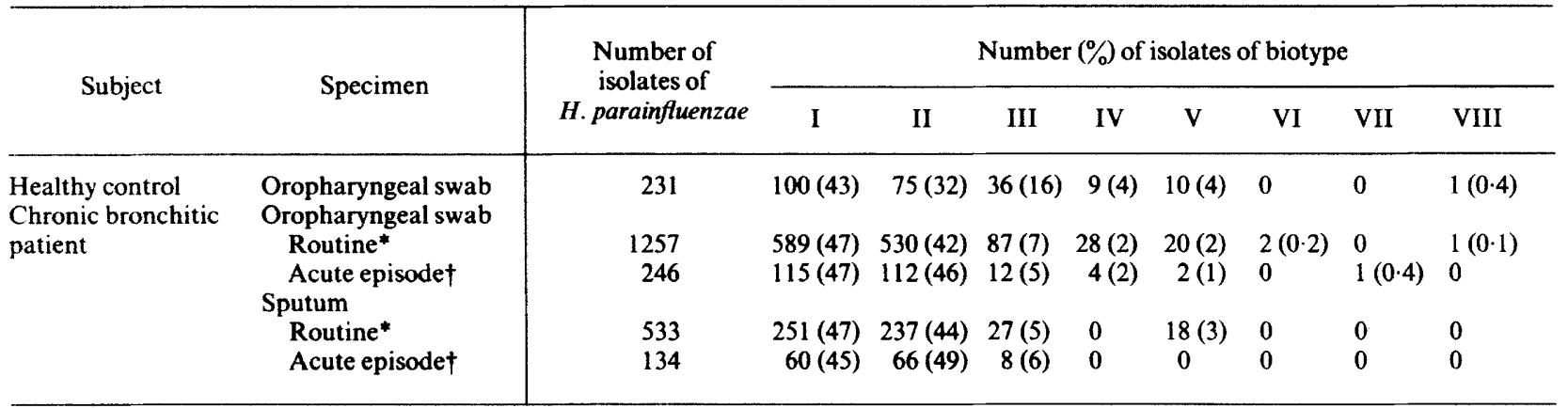

* Routine sample taken in absence of acute exacerbation.

† Sample taken during acute exacerbation. 
isolation rates from oropharyngeal and sputum specimens in the chronic bronchitic population. Little difference was observed in the frequencies of isolation in the presence or absence of acute bronchitis. Biotypes IV-VIII were isolated at a much lower frequency.

We report $50 \mathrm{~V}$-dependent isolates which did not produce indole, urease or ornithine decarboxylase. Acid was produced from glucose, sucrose, mannose and galactose but not from lactose, xylose or mannitol. The isolates were oxidase positive, and the catalase reaction was variable; no haemolysis was observed. We propose that these 50 isolates be classified as $H$. parainfluenzae biotype $\mathrm{V}$. This group of bacteria accounted for c. $2 \%$ of oropharyngeal isolates and $3 \%$ of sputum isolates.

\section{Discussion}

In 1976, in a comprehensive study which included 125 strains of $H$. parainfluenzae, Kilian ${ }^{17}$ proposed a classification of the genus Haemophilus in which strains of $H$. parainfluenzae were assigned to one of three biotypes (I-III) by differences in their production of indole, urease and ornithine decarboxylase. These isolates were mainly from the oral cavities and throats of healthy persons. In 1979, Oberhofer and Back ${ }^{10}$ described two groups of isolates that could not be classified by the Kilian system. They tentatively assigned to a biotype IV seven strains of $H$. parainfluenzae that did not produce indole, urease or ornithine decarboxylase, and they suggested a fifth biotype for three isolates that gave positive results in all three tests. However, in their ensuing discussion, they disclosed that the tentative fifth biotype was actually a mixture of $H$. parainfluenzae and $H$. influenzae.

Frequently, authors fail to include sufficient characteristics to verify that their strains belong to the named species of the Haemophilus genus. In a recent definition of the genus, ${ }^{21}$ the main criterion for placing an aerobic or facultatively anaerobic gram-negative coccobacillus or rod in that genus is a requirement for one or both of the growth factors $\mathrm{X}$ and $\mathrm{V}$. The taxonomically important character distinguishing $H$. parainfluenzae from $H$. influenzae is the capacity to synthesise porphyrin. ${ }^{16}$ The differentiation of Haemophilus spp. by $\mathrm{X}$ and $\mathrm{V}$ disks, as used by Oberhofer and Back, has been noted by several investigators to be misleading ${ }^{22,23}$ at least $12 \%$ of strains of $\mathrm{H}$. influenzae may be mis-identified as $H$. parainfluenzae. ${ }^{24}$ Moreover, the biochemical methods employed do not allow differentiation of Oberhofer and Back's $H$. parainfluenzae biotype IV from other V-dependent species such as $H$. segnis, which also does not produce indole, urease or ornithine decarboxylase. Their seven isolates were oxidase negative but, according to Kilian, ${ }^{17} \mathrm{H}$. parainfluenzae is oxidase positive. This throws doubt on the interpretation of their data and on their biotype classification. In 1984, Brunn et al. ${ }^{5}$ described an isolate of $H$. parainfluenzae biotype IV, which gave positive results in the indole, urease, and ornithine decarboxylase tests and was considered to be the aetiological agent in a patient with bacteraemia. More recently, $H$. parainfluenzae biotypes VI, VII and VIII have been reported ${ }^{25,26}$ among isolates from the genital and respiratory tracts.

In the present study, those isolates that were Vdependent and $\mathrm{X}$-independent were biotyped. Among 2401 such isolates, 2351 were allocated to one of the seven $H$. parainfluenzae biotypes, other than biotype $\mathrm{V}$, as listed in table I. Biotypes I, II and III in the sputum specimens of the bronchitics were also found with similar frequency in the oropharynx: biotypes I and II together constituted $c .90 \%$ of the $H$. parainfluenzae isolates while biotype III was isolated less frequently $(c .6 \%)$. These findings suggest that there may be free exchange of these three biotypes between the URT and LRT, in contrast with our findings for $H$. influenzae. ${ }^{27} \mathrm{H}$. parainfluenzae biotypes I and II were isolated from the oropharynx of control subjects at a similar frequency to that of the chronic bronchitics, though the proportion of biotype II was somewhat greater in cases than in controls, especially during acute episodes. Although comparatively rare, biotype III was cultured approximately twice as often from the oropharynx of the controls as from patients $(\mathrm{p}<$ 0.01 ). The remainder of the 2351 isolates were distributed among biotypes IV, VI, VII and VIII, and these were isolated only from the oropharynx. Consistent with our findings, other investigators have reported that biotypes I-III predominated in both the URT and LRT, though the dominant biotype depended on the age and health of the group studied. ${ }^{10,25,26,28,29}$

The $50 \mathrm{~V}$-dependent isolates which were oxidasepositive but did not produce indole, urease or ornithine decarboxylase were classified as $H$. parainfluenzae biotype V (table I). This biotype was cultured from oropharyngeal swabs of the controls and bronchitics as well as the sputum specimens of the latter group. It was differentiated biochemically from other V-dependent strains of Haemophilus found in the respiratory tract which also do not produce indole, urease or ornithine decarboxylase- $-H$. segnis and $H$. paraphrophilus. The species $H$. parainfluenzae ferments glucose, sucrose, mannose and galactose but not lactose, xylose or mannitol; and it is oxidase-positive. In contrast, $H$. segnis does not ferment mannose and is oxidasenegative; $\boldsymbol{H}$. paraphrophilus does not ferment galactose but does produce acid from lactose. Kilian and Biberstein $^{21}$ consider lactose fermentation to be a reliable criterion to differentiate $H$. paraphrophilus from $H$.parainfluenzae, regardless of $\mathrm{CO}_{2}$ requirement. However, it has been reported that $H$. paraphrophilus is commonly misidentified as $H$. parainfluenzae, ${ }^{30}$ and that at least $83 \%$ of strains submitted to the Centers for Disease Control were identified incorrectly. ${ }^{31}$

In conclusion, $H$. parainfluenzae seems to be part of the indigenous respiratory microflora and has little role in the pathogenesis of chronic bronchitis. 


\section{References}

1. Black CT, Kupferschmid JP, West KW, Grosfeld JL. Haemophilus parainfluenzae infections in children, with the report of a unique case. Rev Infect Dis 1988; 10: 342-346.

2. Albritton WL. Infections due to Haemophilus species other than H. influenzae. Annu Rev Microbiol 1982; 36: 199-216.

3. Cooney TG, Harwood BR, Meisner DJ, Haemophilus parainfluenzae thoracic emphysema. Arch Intern Med 1981; 141: 940-941.

4. Oill PA, Chow AW, Guze LB. Adult bacteremic Haemophilus parainfluenzae infections. Arch Intern Med 1979; 139: 985988.

5. Brunn B, Christensen JJ, Kilian M. Bacteremia caused by a beta-lactamase producing Haemophilus parainfluenzae strain of a new biotype. APMIS Sect B 1984; 92 : 135-138.

6. Frederiksen W, Kilian M. Haemophilus-Pasteurella-Actinobacillus: their significance in human medicine. In: Kilian M, Frederiksen W, Biberstein EL (eds) Haemophilus, Pasteurella and Actinobacillus. London, Academic Press. $1981: 39-55$.

7. Kilian M, Sørensen I, Frederiksen W. Biochemical characteristics of 130 recent isolates from Haemophilus influenzae meningitis. J Clin Microbiol 1979; 9: 409-417.

8. Kuklinska D, Kilian M. Relative proportions of Haemophilus species in the throat of healthy children and adults. Eur $J$ Clin Microbiol 1984; 3: 249-252.

9. Rhind GB, Gould GA, Ahmad F, Croughan MJ, Calder MA Haemophilus parainfluenzae and $H$. influenzae respiratory infections: comparison of clinical features. $\mathrm{Br} \mathrm{Med} J$ 1985; 291: $707-708$.

10. Oberhofer TR, Back AE. Biotypes of Haemophilus encountered in clinical laboratories. J Clin Microbiol $1979 ; 10: 168-174$

11. Roberts DE, Higgs E, Cole PJ. Selective medium that distinguishes Haemophilus influenzae from Haemophilus parainfluenzae in clinical specimens: its value in investigating respiratory sepsis. J Clin Pathol 1987; 40: 75-76.

12. Smith CB, Golden CA, Kanner RE, Renzetti AD. Haemophilus influenzae and Haemophilus parainfluenzae in chronic obstructive pulmonary disease. Lancet 1976; 1 : 1253-1255.

13. Wilson R, Pitt T, Rutman A, Roberts D, Cole P. Haemophilus influenzae and $H$. parainfluenzae slow and disorganise the beating of human cilia in vitro. Clin Sci 1986; 70 Suppl 13: Abstract no. 26.

14. American Thoracic Society: Definitions and classification of chronic bronchitis, asthma, and pulmonary emphysema : a statement by the Committee on Diagnostic Standards for Nontuberculous Respiratory Diseases. Am Rev Respir Dis $1962 ; 85 ; 762-768$.

15. Courcol RJ, Damien JM, Ramon P, Voisin C, Martin GR Presence of alveolar macrophages as a criterion for determining the suitability of sputum specimens for bacterial culture. Eur J Clin Microbiol 1984; 3: 122-125.

16. Kilian M. A rapid method for the differentiation of Haemophilus strains. The porphyrin test. Acta Path Microbiol Scand Sect B 1974; 82: 835-842.

17. Kilian M. A taxonomic study of the genus Haemophilus, with the proposal of a new species. J Gen Microbiol 1976; 93: 962.

18. Back AE, Oberhofer TR. Use of the Minitek system for biotyping Haemophilus species. J Clin Microbiol 1978; 7 : 312-313.

19. Kawakami Y, Okimura Y, Kanai M. Biochemical characterization of Haemophilus species with the Minitek differentiation system. J Clin Microbiol 1981; 14: 579-581.

20. Steel KJ. The oxidase reaction as a taxonomic tool. $J$ Gen Microbiol 1961; 25: 297-306.

21. Kilian M, Biberstein EL. Genus II: Haemophilus. In: Kreig NR, Hold JG (eds) Bergey's Manual of systematic bacteriology, vol 1. Baltimore, Williams and Wilkins. 1984: 558-569.

22. Evans NM, Smith DD. The effect of the medium and source of growth factors on the satellitism test for Haemophilus species. J Med Microbiol 1982; 5: 509-514.

23. Kilian M. Haemophilus. In: Lennette EH, Balows A, Hausler WJ, Truant JP (eds) Manual of clinical microbiology, 3rd edn. Washington, DC, American Society for Microbiology. 1980: 330-336.

24. Kilian M. The Genus Haemophilus. In: Starr MP, Stolp H, Trüper HG, Balows A, Schleyel HG (eds) The prokaryotes. $A$ handbook on habitats, isolation and identification of bacteria. Berlin, Springer-Verlag. 1981 : 1371-1382.

25. Doern GV, Chapin KC. Determination of biotypes of Haemophilus influenzae and Haemophilus parainfluenzae. A comparison of methods and a description of a new biotype (VIII) of Haemophilus parainfluenzae. Diagn Microbiol Infect Dis 1987; 7: 269-272.

26. Sturm AW. Isolation of Haemophilus influenzae and Haemophilus parainfluenzae from genital-tract specimens with a selective culture medium. J Med Microbiol 1986; 21 : 349-352.

27. Butt HL, Clancy RL, Cripps AW et al. Bacterial colonisation of the respiratory tract in chronic bronchitis. Aust $N Z J$ Med 1990; 20: 35-38.

28. Watson KC, Kerr EJC, Hinks CA. Distribution of biotypes of Haemophilus influenzae and $H$. parainfluenzae in patients with cystic fibrosis. J Clin Pathol 1985; 38: 750-753.

29. Houang E, Philippou M, Ahmet Z. Comparison of genital and respiratory carriage of Haemophilus parainfluenzae in men. J Med Microbiol 1989; 28: 119-123.

30. Albritton WL. Species identification in Haemophilus infection. Rev Infect Dis 1988; 10: 1226.

31. Jones RN, Slepack J, Bigelow J. Ampicillin-resistant Haemophilus paraphrophilus laryngo-epiglottitis. J Clin Microbiol 1976; 4 : 405-407. 